\title{
Progress in the clinical development and utilization of vision prostheses: an update
}

This article was published in the following Dove Press journal:

Eye and Brain

II May 2016

Number of times this article has been viewed

\section{Alice Brandli \\ Chi D Luu \\ Robyn H Guymer \\ Lauren N Ayton}

Centre for Eye Research

Australia, Department of Surgery (Ophthalmology), The University of Melbourne, Royal Victorian Eye and Ear Hospital, Melbourne, VIC, Australia
Correspondence: Lauren N Ayton Centre for Eye Research Australia, Department of Surgery (Ophthalmology), The University of Melbourne, Royal Victorian Eye and Ear Hospital, Level I, 32 Gisborne St, East Melbourne, VIC 3002, Australia

Tel +6I 399298066

Email Inayton@unimelb.edu.au

\begin{abstract}
Vision prostheses, or "bionic eyes", are implantable medical bionic devices with the potential to restore rudimentary sight to people with profound vision loss or blindness. In the past two decades, this field has rapidly progressed, and there are now two commercially available retinal prostheses in the US and Europe, and a number of next-generation devices in development. This review provides an update on the development of these devices and a discussion on the future directions for the field.
\end{abstract}

Keywords: vision prostheses, bionic eye, vision restoration, blindness, medical bionics, retinitis pigmentosa

\section{Introduction}

Loss of sight and blindness can result from pathological changes affecting the visual system at different locations, including the retina, optic nerve, posterior visual pathway, and visual cortex, and it is estimated that over 285 million people worldwide have vision impairment. ${ }^{1}$ The physical, emotional, and social implications of vision loss are significant, and hence, numerous international research teams are working toward the development of interventions that would restore vision. One such intervention uses vision prostheses, which are implantable devices that use electrical currents to stimulate surviving retinal or cortical neurons to produce light percepts.

Vision prostheses were first investigated in the 1930s by the German ophthalmologist Carl Foerster, who discovered that direct electrical stimulation of the visual cortex enabled a blind patient to perceive light. ${ }^{2}$ From this early beginning, the development of vision prostheses has progressed to a point where there are now two commercially available devices in the US and Europe, with a large range of next-generation devices in development. A map of the currently active vision prosthesis research groups is shown in Figure 1.

The normal course of visual perception begins with light passing through the cornea and lens to be focused on the retina. The photoreceptor cells preferentially absorb visible light to trigger phototransduction, a process that converts light into an electrical signal. The electrical signal produced is processed by interneurons (bipolar cells, horizontal cells, and amacrine cells) and sent by the retinal ganglion cells to the lateral geniculate nucleus by way of the optic nerve and then onto the visual cortex. The sum total of visual processing from light absorption 


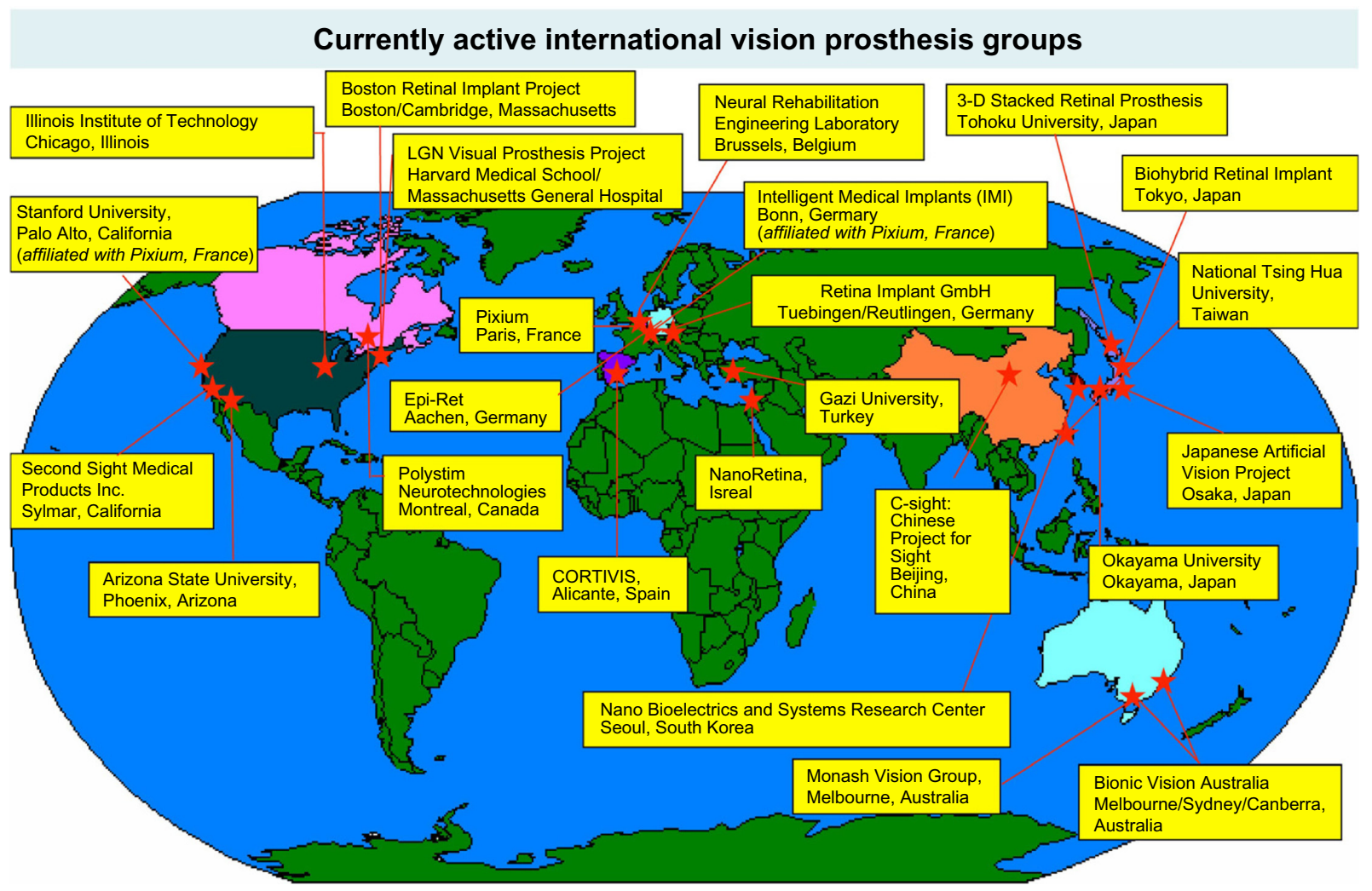

Figure I Currently active vision prosthesis research groups, as of January 2016.

Notes: Courtesy of Professor Joe Rizzo, Dr Lauren Ayton, and the Detroit Institute of Ophthalmology.

to cortical processing is an image complete with color, spatial, edge, and motion information.

Vision prostheses can be implanted in a number of locations in this visual pathway, depending on the cause of vision loss. Devices have been designed for the retina (either epiretinal, ${ }^{3}$ subretinal, ${ }^{4}$ or suprachoroidal),${ }^{5}$ for implantation within a scleral pocket, ${ }^{6}$ cuffs that encircle the optic nerve, ${ }^{7}$ or cortical implants which directly stimulate the visual cortex $^{8}$ (Figure 2). This review will provide an overview of the basic science behind vision prostheses and an update on the progress of current technologies.

\section{Visual prostheses: from phosphene to visual scene}

Classically, light perception begins with the photoreceptors converting light into an electrical signal in the retina, which travels by the visual pathway to the visual cortex, where the interpretation of these signals gives us our vision. In the absence of a viable visual pathway, or if no light stimulus is present, visual percepts are still possible, and are known as "phosphenes". Phosphenes appear independently of light stimuli and have been described as sparks of light that can appear white or colored, but also with a structured appearance (ie, during migraine auras). Phosphenes can appear spontaneously, such as during periods of visual deprivation, or can be induced from magnetic, electrical, or mechanical stimulation.

The production of phosphenes by electrical stimulation forms the basis of visual prosthetic technology. The ability of electrical stimulation to produce phosphenes has long been recognized, with Volta first describing the visual percepts created from electrical stimulation of the retina over 200 years ago. In the past 25 years, however, the controlled production of phosphenes has been exploited for the purposes of visual prostheses. ${ }^{9}$ Phosphenes elicited from electrical stimulation are typically reported as white flashes of light with welldefined shapes. ${ }^{10}$ Importantly, the visual characteristics of electrically evoked phosphenes, such as brightness, size, shape, and location, can be manipulated by altering electrical stimulation parameters..$^{10}$ For example, increasing the charge of electrical stimulation creates larger and brighter phosphenes. ${ }^{10}$

Controlled presentation of phosphenes forms the building blocks of visual scenes, with electrical stimulation producing 


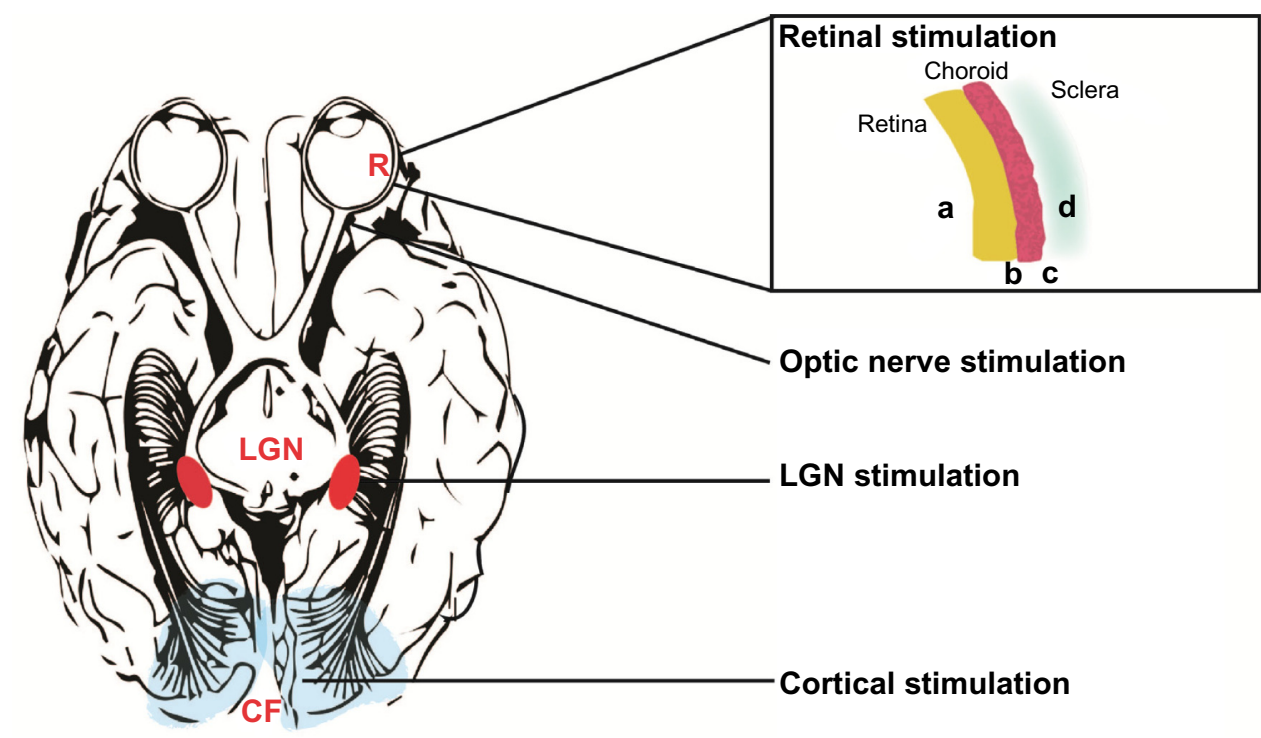

Figure 2 Locations of vision prostheses.

Notes: A schematic of the visual pathway as drawn from the ventral side of the brain with the inset box depicting the retinal sites of visual prosthesis implants.Visual prosthesis devices have been implanted at the following locations: within the eye and retina ( $R$ ) at a, epiretinal; b, subretinal; c, suprachoroidal; and d, intrascleral sites, the optic nerve, the LGN and within the visual cortex, adjacent to the CF. Electrical stimulation at these sites elicits phosphenes that can be used to create low-resolution vision.

Abbreviations: CF, calcarine fissure; LGN, lateral geniculate nucleus; R, retina.

visual percepts that are capable of interacting with each other to form images (Figure 3). ${ }^{11,12}$ Intensive psychophysical testing and training is required to teach patients to interpret phosphene images and, depending on the subject, these organized phosphene maps provide varying levels of rudimentary gray-scale vision. The vision currently achievable with vision prostheses is termed "ultra-low vision", ${ }^{13}$ with the best-performing subjects able to perceive large high-contrast shapes, recognize very large letters, and/or detect slow moving objects. ${ }^{3,5,14,15}$ The best visual acuity that has been reported



B

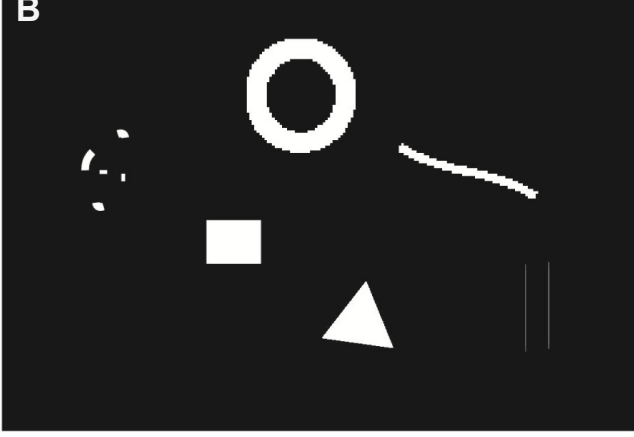

C

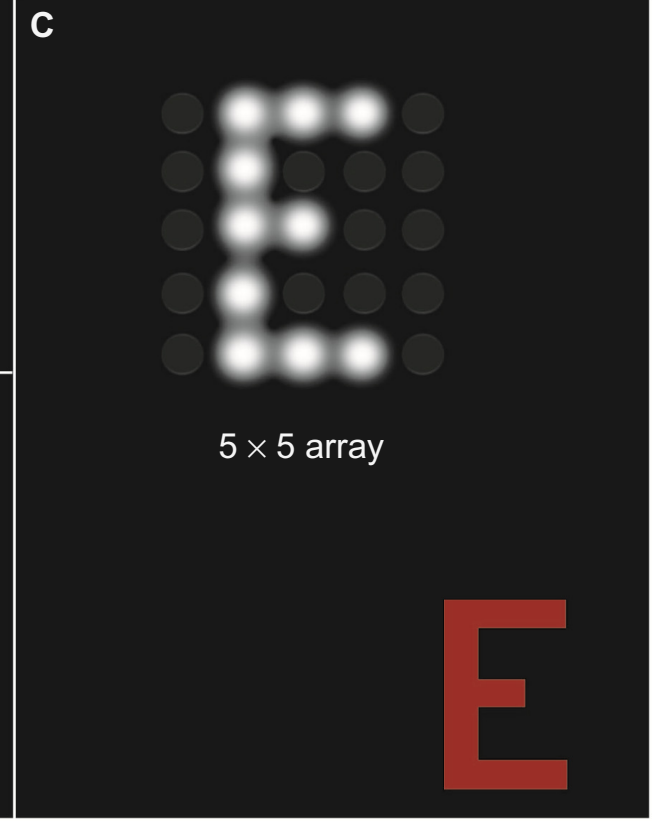

Figure 3 Examples of the simulated phosphenes (single and patterned) and their characteristics as reported by patients.

Notes: The theoretically expected phosphene from electrical stimulation of the visual pathway is a white (colorless) circle (A). Other shapes have been reported, such as dots, donut-shaped circles, lines, squares, triangles, and matchsticks (B). Patterns of phosphenes elicited can form an image (the letter E), as depicted on a $5 \times 5$ electrode array (C). The stimulus, a red $\mathrm{E}$, is shown in the lower corner. 
to date is with a subretinal vision prosthesis, which improved the acuity of a subject from bare light perception to 20/546 $(\log$ MAR 1.43$){ }^{16}$

\section{Basic architecture of visual prosthetic systems}

While all vision prostheses incorporate different technologies and stimulation strategies, the general principles are constant. The devices convert a visual image into electrical stimulation by two mechanisms: "classical visual prostheses" and "optical sensor prostheses".

Classical prostheses (Figure 4A) comprise a camera to capture the visual scene and a vision processor to externally
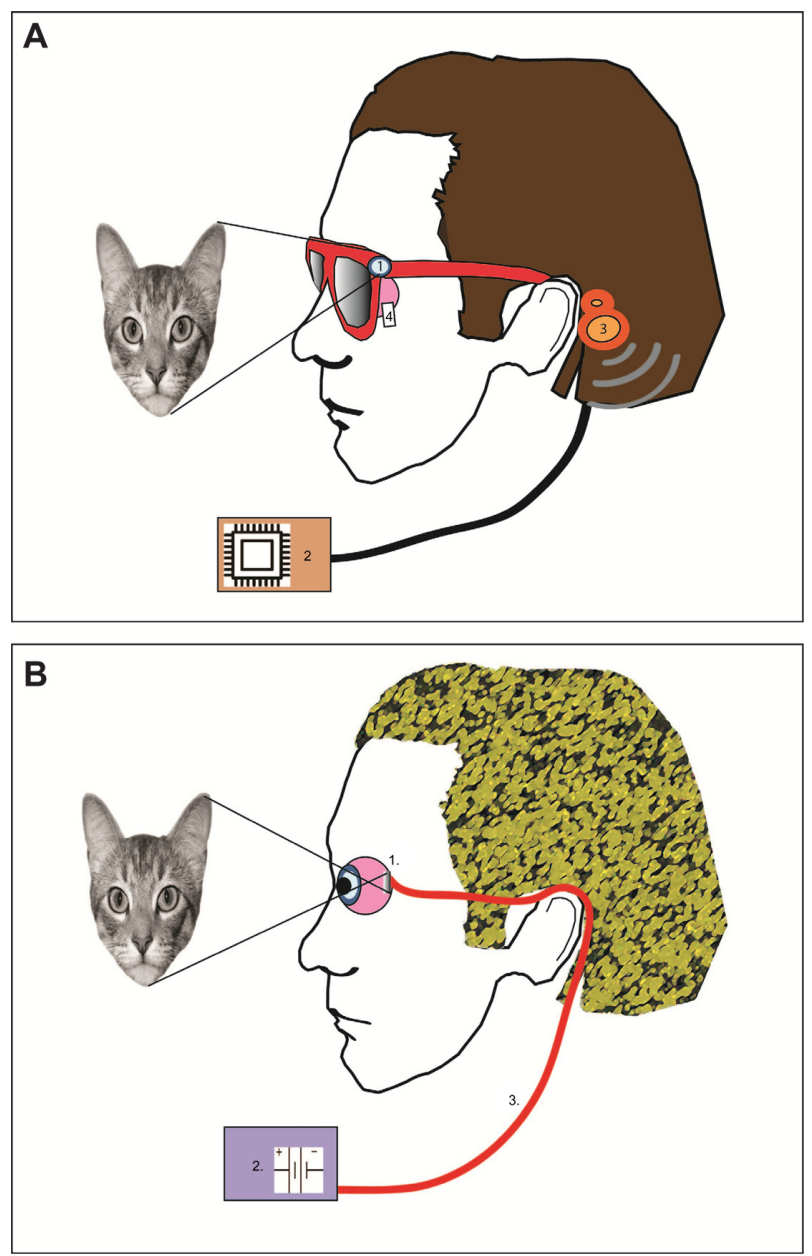

Figure 4 Schematic of a classical prosthesis with a retinal implant (A) and optical sensor prosthesis (B).

Notes: Classical prosthesis requires a camera ( $\mathrm{I}$ ) to capture images. The image (a cat) is then processed externally (2) and converted to an electrical stimulation pattern that is transmitted by a wireless receiver (3) to the retinal implant (4) to elicit phosphenes. Optical sensor prosthesis relies on image capture by a subretinally implanted array (I). The light signal is amplified by power derived from an external battery source (2) that is connected using a cable (3). The multiphotodiode array is able to elicit phosphenes within the retina without the use of a camera and visual processing package. convert images into electrical waveforms, which are then sent to an implanted array of electrodes to stimulate visual pathway neurons. Communication between the camera, the vision processor, and the electrode array will require implantation of either a wireless (ie, telemetry) or wired link. The only currently commercially available camera-based classical retinal prosthesis is Argus II epiretinal device (Second Sight Medical Products, Inc., Sylmar, CA, USA). ${ }^{3}$

Optical sensor prostheses (Figure 4B) are comparable to the function of photoreceptors, using photodiode technology that converts visible light into electrical activity without the need for an external camera. Optical sensors are composed of a micro-photodiode array that is capable of converting visible light into electrical currents to directly stimulate the retinal ganglion cells. These devices make use of the patient's own optical system (cornea, iris, and lens) to focus light onto the micro-photodiode array. ${ }^{17}$ Optical sensor prostheses allow natural eye movements (without the need to direct a video camera to the object of interest) and tend to be more compact, but require relatively high levels of light to work effectively. In addition, optical sensor prostheses do not have the capacity to optimize stimulation parameters by processing of the external camera image, meaning that the device is much more reliant on the qualities of the natural visual scene. The only currently commercially available optical sensor-based retinal prosthesis is Alpha IMS subretinal device (Retina Implant AG, Reutlingen, Germany). ${ }^{4}$

Images of the commercially available and prototype devices can be found in previously published clinical trial reports on Argus II (Second Sight Medical Products, Inc.), ${ }^{18}$ Alpha IMS (Retina Implant AG), ${ }^{14}$ and 24-channel suprachoroidal device (Bionic Vision Australia, Parkville, Victoria, Australia). ${ }^{5}$

\section{Clinical trial outcomes of current vision prostheses}

The electrode or photodiode array of a visual prosthesis can be implanted at various sites along the visual pathway. The efficacy of such implants to produce visual phosphenes has been shown in the retinal, optic nerve, and cortical locations in previous clinical trials. There are a number of advantages and disadvantages with each surgical location, as outlined in Table 1. A summary of the chronic clinical trials completed to date is shown in Table 1.

\section{Retinal prostheses}

Retinal prostheses are designed to restore vision to people who have lost photoreceptor function from retinal dystrophies 
Table I Summary of the vision prosthesis clinical trials completed to date

\begin{tabular}{|c|c|c|c|c|c|c|c|}
\hline $\begin{array}{l}\text { Name of } \\
\text { device }\end{array}$ & $\begin{array}{l}\text { Implant } \\
\text { location }\end{array}$ & $\begin{array}{l}\text { Classical camera } \\
\text { based or optical } \\
\text { sensor based }\end{array}$ & $\begin{array}{l}\text { Number of } \\
\text { stimulating } \\
\text { electrodes }\end{array}$ & $\begin{array}{l}\text { Patients } \\
\text { enrolled }\end{array}$ & $\begin{array}{l}\text { Cause of } \\
\text { patient's } \\
\text { vision loss }\end{array}$ & $\begin{array}{l}\text { Visual performance } \\
\text { outcomes assessed }\end{array}$ & References \\
\hline Argus II & Epiretinal & Camera based & 60 & 30 & $\begin{array}{l}\mathrm{RP}, \\
\text { choroideremia }\end{array}$ & $\begin{array}{l}\text { Phosphene descriptions, } \\
\text { square localization, direction } \\
\text { of motion, grating VA, letter } \\
\text { reading, ADL, FLORA }\end{array}$ & $2,15,18,19$ \\
\hline Argus I & Epiretinal & Camera based & 16 & 6 & RP & Phosphene descriptions & 20,21 \\
\hline Epiret 3 & Epiretinal & Camera based & 25 & 6 & RP & $\begin{array}{l}\text { Phosphene descriptions, NEI- } \\
\text { VFQ-25 }\end{array}$ & 22,23 \\
\hline IMI & Epiretinal & Camera based & 49 & 20 & $\begin{array}{l}\text { RP, Usher's } \\
\text { syndrome }\end{array}$ & Phosphene descriptions & 24 \\
\hline Alpha IMS & Subretinal & Optical sensor & $\mathrm{I}, 500$ & $\begin{array}{l}3,9,29 \\
\text { (three } \\
\text { reported } \\
\text { trials) }\end{array}$ & RP & $\begin{array}{l}\text { BALM, BAGA, grating VA, } \\
\text { Landolt C-rings (FRACT) VA, } \\
\text { object identification, letter } \\
\text { reading, clock reading, gray- } \\
\text { level discrimination, ADL, } \\
\text { patient reports }\end{array}$ & $14,16,25,26$ \\
\hline $\begin{array}{l}\text { ASR } \\
\text { microchip }\end{array}$ & Subretinal & Optical sensor & 5,000 & 6 & RP & $\begin{array}{l}\text { ETDRS VA, Humphreys } \\
\text { perimetry, ERG, patient } \\
\text { reports }\end{array}$ & 27 \\
\hline $\begin{array}{l}\text { STS } \\
\text { prosthesis }\end{array}$ & Intrascleral & Camera based & 49 & 2 & RP & $\begin{array}{l}\text { Phosphene descriptions, } \\
\text { object detection and } \\
\text { discrimination, detection of } \\
\text { motion, grasping object }\end{array}$ & 5,28 \\
\hline $\begin{array}{l}\text { BVA } 24 \\
\text { channel } \\
\text { device }\end{array}$ & Suprachoroidal & Camera based & 24 & 3 & RP & $\begin{array}{l}\text { BALM, Landolt C-rings } \\
\text { (FRACT) VA }\end{array}$ & 29 \\
\hline AV-DONE & Optic nerve & Camera based & 7 & I & RP & Phosphene descriptions & 30 \\
\hline MiViP & Optic nerve & Camera based & 4 & $\begin{array}{l}\text { I, } 2 \text { (two } \\
\text { reported } \\
\text { trials) }\end{array}$ & RP & $\begin{array}{l}\text { Phosphene descriptions, } \\
\text { object recognition, VEP }\end{array}$ & 6,31 \\
\hline $\begin{array}{l}\text { Dobelle } \\
\text { implant }\end{array}$ & Visual cortex & Camera based & 64 & I & Trauma & $\begin{array}{l}\text { Landolt C-rings, Snellen } \\
\text { tumbling E }\end{array}$ & 32 \\
\hline
\end{tabular}

Abbreviations: ADL, activities of daily living; Alpha IMS, Intelligent Medical Systems; AV-DONE, Artificial Vision by Direct Optic Nerve electrode; BAGA, basic grating acuity test; BALM, basic light and motion test; BVA, Bionic Vision Australia; ERG, electroretinogram; ETDRS, Early Treatment Diabetic Retinopathy Study; FLORA, functional low-vision observer-rated assessment; FRACT, Freiburg acuity and contrast test; IMI; intelligent medical implant; MiViP, Microsystems-Based Visual Prosthesis for Optic Nerve; NEI-VFQ-25, National Eye Institute Visual Function Questionnaire-25; RP, retinitis pigmentosa; STS, suprachoroidal-transretinal stimulation; VA, visual acuity; VEP, visually evoked potential.

such as retinitis pigmentosa (RP) and age-related macular degeneration (AMD). Such photoreceptor dystrophies are the leading causes of blindness in working adults ${ }^{19}$ and result in vision loss due to the permanent loss of photoreceptors. Despite the loss of photoreceptors, the inner retina and posterior visual pathway remain relatively intact and connected to the higher visual processing region of the brain. ${ }^{20,21}$ To date, regulatory approval for retinal prostheses has only been received for patients with profound vision loss (such as in RP or choroideremia), but recent trials have commenced with a small cohort of patients with severe geographic atrophy from AMD.

The main limitation with the retinal location is that subjects must have an intact inner retina and posterior visual pathway (optic nerve to visual cortex) for the devices to work.
As such, retinal prostheses will only be suitable for people who have pathology limited to the outer retina.

There are four potential locations for a vision prosthesis at or near the retina. Devices can be implanted onto the inner retina (epiretinal), ${ }^{3}$ between the retina and the choroid (subretinal), ${ }^{4}$ behind the choroid (suprachoroidal), ${ }^{5}$ or within a pocket in the sclera (transscleral). ${ }^{6}$

As the aim of retinal prostheses is to stimulate the inner retinal neurons (mainly the retinal ganglion cells), many groups have investigated the use of an epiretinal device, which is positioned closest to the inner retinal neurons using retinal tacks. Its close apposition to the target neurons means that it requires lower currents to cause retinal ganglion cell activation. ${ }^{22}$ However, this location is a more challenging surgical location than the more posterior locations (suprachoroidal 
and transscleral), leading to a higher incidence of surgical and device-related adverse events. ${ }^{5}$

At this time, four medical device companies are undergoing clinical trials of retinal prostheses, and two of these have regulatory approval to sell in the US and Europe (Second Sight Medical Products, Inc.'s Argus II epiretinal and Retina Implant AG's subretinal devices). In all the clinical trials detailed, subjects had profound vision loss from RP or choroideremia (of bare light perception or worse) at baseline and were implanted with prototype devices. Postmarket data on the commercially available devices is expected in the near future.

There are also a number of other groups that have completed previous clinical trials or are expected to begin testing in the near future. For brevity, this review has focused on the four groups with the most recent clinical results, and a more thorough summary of other work can be found in previous reviews. ${ }^{23-25}$

\section{Second Sight Medical Products, Inc.'s Argus II epiretinal device (CE mark and US Food and Drug Administration approval)}

The Argus II (Second Sight Medical Products, Inc.) contains 60 electrodes organized on a $6 \times 10$ array, which is implanted epiretinally. The electrode array is wirelessly powered and the electronic processing unit is mounted externally to the sclera. Patient trials commenced in 2007, with 30 RP or choroideremia patients enrolled by 2009 . The results of these trials were first reported in 2012, ${ }^{18}$ and long-term results (up to 3 years) were published in $2015 .^{3}$ Argus II was the first vision prosthesis device to receive both CE mark and US Food and Drug Administration regulatory approval, and is now undergoing postmarket surveillance studies.

The initial clinical results for Argus II demonstrated that phosphenes were able to be detected in all 30 subjects. Visual acuity was approximated using a four-alternative forced choice measure of grating acuity, and seven patients completed the task, giving acuity measures of between 2.9 and $1.6 \log$ MAR. ${ }^{18}$ With the device on, most subjects $(27 / 28)$ performed better in detecting a white square on a black computer screen (square localization task) and 16 of 28 subjects could detect motion on a computer-based task. ${ }^{18}$ This study also found that six of the subjects were able to identify relatively small alphabet letters (the smallest measuring $0.9 \mathrm{~cm}$ at $30 \mathrm{~cm}$ ) and four subjects could correctly identify unrehearsed words of between two and four letters. ${ }^{15}$ Orientation and mobility (O\&M) performance was also assessed through two novel measures: the find the door test and follow the line. At 6 months postimplantation, $86 \%$ of participants showed improvement in find the door task and $73 \%$ improved in following the line task with the device on. ${ }^{26}$

Long-term results from a multicenter clinical trial of Argus II were published in 2015, and showed that at 3 years postimplantation, visual performance was stable for square localization ( $94 \%$ at 1 year vs $89 \%$ at 3 years), direction of motion ( $62.5 \%$ at 1 year vs $55.6 \%$ at 3 years), find the door (53\% at 1 year vs $54 \%$ at 3 years), and follow the line $(73 \%$ at 1 year vs at $68 \% 3$ years) tasks. ${ }^{3}$ The Argus II clinical trials have also included a new functional low-vision observerrated assessment, which combines visual tasks scoring (eg, mobility tasks and activities of daily living), self-reported user experience, and case-study assessment (a modified quality of life questionnaire $)^{27}$ by a rehabilitation expert, which showed $80 \%$ of patients reported that the device had a positive impact on their daily life at the 1-year follow-up. ${ }^{3}$

In 2015, Second Sight Medical Products, Inc. expanded the indications for Argus II prosthesis by implanting in a patient with vision loss caused by atrophic AMD. Vision loss is less severe in end-stage atrophic AMD, compared to people with end-stage RP (which have been the main recipients of implants to date). In RP, a widespread loss of central and peripheral vision is expected, whereas in AMD, it is only central vision loss which occurs with maintenance of peripheral vision. It is unknown what effects the epiretinal implant surgery will have on the normal peripheral vision in subjects with AMD. Another five atrophic AMD patients are expected to be implanted with the Argus II device in the near future. The outcomes for this study will be eagerly awaited.

\section{Retina Implant AG's Alpha IMS subretinal device (CE mark approval)}

The Alpha IMS subretinal device (Retina Implant AG) is the first regulatory-approved optical sensor-based vision prosthesis. The device consists of an array of 1,500 photodiodes, which is implanted between the retinal pigment epithelium and the neurosensory retina. Due to the use of optical sensor design, the Alpha IMS does not use an external camera. The Alpha IMS device received CE mark regulatory approval in 2013.

Originally, the photodiodes used in these implants were passive and relied on incidental light to power the electrodes; but this incidental light was found to be insufficient to activate the retinal ganglion cells in degenerated retinae. ${ }^{28,29}$ Hence, the current Alpha IMS device now couples 1,500 photodiodes 
to an external power source (implanted behind the ear) that amplifies the stimulation into a usable current to stimulate

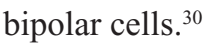

An initial clinical study of the Alpha IMS was conducted in nine subjects with RP and each subject was followed for 9 months. The results of the study showed that eight of the nine subjects were able to perceive phosphenes with the device. ${ }^{31}$ Light localization was possible in $7 / 9$ subjects, and motion detection in 5/9 subjects. Three of the subjects were able to read large letters, and two were able to complete the Landolt $\mathrm{C}$ letter optotype recognition task, with acuities of up to $1.4 \log$ MAR recorded.

In 2015, the results of a larger 12-month multicenter trial $(\mathrm{N}=29)$ were reported for the Alpha IMS device. ${ }^{14}$ This study found that the device improved the detection, localization, and recognition of geometric shapes, with four of the subjects able to read large letters. In terms of their daily activities, eight subjects could localize objects but not recognize details, 13 reported being able to see shapes and details, and eight found no improvement in object detection. A significant restoration of "visual function which they use in daily life" was reported in 13 of the 29 subjects. Measurable grating acuity was up to 3.3 cycles per degree, and the best-measured visual acuity (using the Landolt $\mathrm{C}$ optotype) was 20/546.

Interestingly, 27 patients had light perception at 1 month postimplantation; however, this reduced to ten patients at 12 months postimplantation, suggesting a decrease in retinal function with time. Similarly, light localization was possible in $40 \%$ of subjects at 1 month postimplantation, but fell to $20 \%$ by 1 year. ${ }^{14}$ Without a randomized trial, it will be difficult to determine if the decline is the expected natural history of the disease or is in some way related to the device.

Postmarket surveillance studies of the Alpha IMS continue.

\section{Pixium Vision's IRIS-I epiretinal device}

The IRIS-I device, manufactured by Pixium Vision (Paris, France), is a 49-electrode wireless epiretinal implant, which was developed from the technology used in the Intelligent Medical Implant device. Acute experiments of the Intelligent Medical Implant lasting 45 minutes in patients with RP showed that 19/20 subjects experienced phosphenes. ${ }^{32}$ A clinical trial was then completed, in which four subjects with RP were implanted for 1 year. ${ }^{33}$ This study showed that the device was well tolerated with good electrode to retina contact and enabled subjects to identify simple patterns (vertical/horizontal bars and a cross).
Pixium Vision's second-generation epiretinal device (the IRIS-II) is composed of 150 electrodes and has recently begun clinical trials in Europe. Pixium Vision is also developing a subretinal optical sensor device that comprises 37 photodiode-amplifier-electrode units. The multiphotodiode array (MDPA) unit, termed "Prima", is anticipated to enter into human trials in 2016.

\section{Bionic Vision Australia's suprachoroidal device}

Bionic Vision Australia and Bionic Vision Technologies (Australia) have developed a suprachoroidal implant, which is placed between the choroid and the sclera. One of the main benefits of this device, despite being located further away from the target ganglion cells, is that the electrode array can be inserted in a minimally invasive and less technically challenging surgical approach than that required for other locations. The increase in distance between the retinal ganglion cells and the suprachoroidal implant location does result in higher electrical currents needing to be delivered to stimulate phosphenes. ${ }^{10}$ However, studies in patients and animal models have demonstrated that the retina can be safely and effectively stimulated with suprachoroidal implants with a relatively good dynamic range. . 34,35

In 2014, the results of the Bionic Vision Australia prototype trial were published using a 24-electrode suprachoroidal device in three subjects with end-stage RP. ${ }^{5}$ The prototype suprachoroidal device was connected to an external percutaneous connector that was implanted behind the ear to allow a wide variety of stimulation parameters to be tested.

The study found that the surgical approach was safe and effective, with no unexpected serious device-related adverse events. Retinal hemorrhages did occur 3-4 days after implantation surgery, but resolved without the need for further treatment. Phosphenes were reported in all subjects, although there was variation in the optimal stimulation parameters for each individual. ${ }^{10}$ Light localization was possible in all patients, with a success rate ranging from $66 \%$ to $97.5 \%$. Visual acuity testing using Landolt $\mathrm{C}$ optotypes was recorded in one subject, with a mean score of $2.62 \log$ MAR. Importantly, the devices remained functional over the 18-month study in all patients.

A second-generation suprachoroidal device has been developed, and clinical trials are planned for late 2016.

\section{Optic nerve prostheses}

Two optic nerve prostheses have been tested in clinical trials to date: the Artificial Vision by Direct Optic Nerve electrode 
(AV-DONE; Japan $)^{36}$ and the Microsystems-Based Visual Prosthesis for Optic Nerve (MiViP; Belgium). ${ }^{37}$ These devices were tested in clinical trials of one and three subjects, respectively, with all of them having profound vision loss from RP.

The AV-DONE device has seven electrodes and is implanted directly onto the optic nerve in the eye itself, which allows for easier surgical access. The device has been implanted into the eye of a subject with RP, and was able to produce phosphenes from five of the seven electrode locations when stimulated at 9 and 25 months postoperatively. ${ }^{36}$ The study also found that there were no severe adverse events in the 25 months after implantation, but did not report on any visual acuity or functional vision outcomes. ${ }^{36}$

The second optic nerve prosthesis that has been tested in human subjects is the MiViP, which is a four-electrode cuff that is implanted around the optic nerve intracranially. ${ }^{37}$ An initial report in 2003 described the initial results in one RP subject, where the device was shown to be able to be safely implanted and produce phosphenes, which could be used to detect simple patterns. ${ }^{37}$ A more recent study of the MiViP in two subjects confirmed that stimulation of the device resulted in measurable cortical evoked potentials, and subjects reported phosphenes within a limited central area of the visual field. ${ }^{7}$

Due to challenges with the surgical location of this device, there has been limited progress with optic nerve prostheses in the past few years.

\section{Cortical prostheses}

It has long been known that phosphenes can also be evoked by direct cortical stimulation. Indeed, single-site electrical stimulation of the visual cortex was one of the first reported techniques to produce visual phosphenes in the early 1900 s. ${ }^{2,38}$ Later studies investigated whether multielectrode devices could be used to produce pattern vision, with a study by Brindley and Lewin ${ }^{39}$ showing that 39 of 80 implanted cortical electrodes produced phosphenes in a patient with glaucoma and retinal detachment. The largest clinical trial to date using cortical prostheses has been the work of William Dobelle $^{40}$ in the early 2000s, when 16 participants were implanted with a cortical implant. Unfortunately, little has been reported about the outcomes of this trial.

Implantation of either surface or penetrating microelectrodes at the visual cortex provides some technical advantages over the retina and optic nerve. First, cortical prostheses could provide vision to patients who are unable to benefit from a retinal implant due to loss of healthy ganglion cells from diseases such as glaucoma, optic atrophy, or ocular trauma. ${ }^{41}$
Second, the visual cortex is retinotopically organized, and foveal retinal ganglion cell projections are magnified over a larger surface area relative to the retina. ${ }^{42,43}$ The larger surface area is ideal for the implantation of multiple stimulating electrodes. Although not yet tested, researchers have suggested that implantation of larger or multiple electrode arrays at the visual cortex could provide higher-resolution artificial vision.

A disadvantage of cortical implants is the surgical location, requiring a significant neurosurgical procedure, with exposure of the dura mater, and the possible need to stimulate deep into the calcarine fissure where the foveal projections are buried. ${ }^{44}$ In addition, implantation requires the permanent attachment of electrode devices, which may risk the normal functionality of the cortex (reviewed by Lewis et al). ${ }^{45}$

Recently, novel cortical prosthetic devices have been developed by a number of research and commercial groups. Preclinical studies by the Monash Vision group in Australia have shown that intracortical penetrating microelectrodes can be safely implanted under chronic settings. ${ }^{46} \mathrm{~A}$ clinical trial of their device, known as the Gennaris, is anticipated in the next year.

A surface cortical electrode array, known as Orion, has also been developed by Second Sight Medical Products, Inc., and clinical trials are anticipated in the near future.

\section{Comparisons between currently available devices and the need for standardized testing}

Visual performance comparisons between the currently available vision prostheses are difficult due to the lack of standardized testing methodology. For example, when comparing visual acuity, the Alpha IMS subretinal implant reports a higher maximum visual acuity score of $1.43 \log$ MAR compared to $1.80 \log$ MAR for the Argus II epiretinal implant, but the methods used to determine acuity are different (Landolt $\mathrm{C}$ letter optotype acuity vs grating acuity, respectively), and hence, direct comparison is not possible.

In addition, visual acuity is a poor predictor of useful vision in the ultra-low vision category. Real-world vision testing uses measurements of activities of daily living or O\&M tasks, and has been found to be more relevant to users of early vision prosthesis technology. ${ }^{27}$ Subjective assessments, such as quality of life questionnaires, seek to understand whether the device has improved the patient's lives and have also been shown to be important for such clinical trials. ${ }^{47,48}$

Caution needs to be taken when interpreting and comparing the results of visual prosthesis clinical trials, as the lack of standardization in ultra-low vision assessment makes direct 
comparisons difficult. New methods of vision functional testing are required, where ideally, testing should include standardized methods for visual acuity grading, object location and detection, O\&M tasks, and the patient's perspective on the implant. A committee called the Harmonization of Outcomes and Vision Endpoints in Vision Restoration Trials Taskforce has been formed to address the need for standardization in the ultra-low vision field, and standards from this working group are anticipated in the near future. ${ }^{49}$

\section{Conclusion and future directions}

The field of vision prostheses has rapidly evolved over the past decade, with two retinal implants now commercially available and a number of next-generation devices in development. The improvements in vision afforded by current devices are still modest, with subjects able to locate and identify large objects and navigate O\&M tasks more effectively, but not read small print or recognize faces. While the former outcomes are significant for people who have baseline vision levels at bare light perception vision or worse, it is certainly the aim that these technologies will be improved with time to allow higher-resolution vision.

This review has provided a brief overview of the various designs of prosthesis available (camera based vs optical sensor based) and the range of surgical locations possible along the visual pathway. This range of methodology is required, as subjects with different causes of vision loss and different levels of residual vision are likely to benefit from different prostheses. Future questions to be addressed include the efficacy of vision prostheses in subjects who have very longstanding blindness (which often leads to retinal and cortical reorganization) or even in those born blind. Both of these situations provide significant challenges due to the disruption or malformation of the visual pathway; however, restoring vision after decades of loss or giving rudimentary vision to someone who has never experienced it would certainly be a triumph for this technology.

Future vision prostheses will likely benefit from improvements in the density and shape of phosphenes, allowing higher-resolution images, and increases in the field of view using the device. This may either be achieved by developments in materials and hardware so that a higher number of electrodes can be incorporated onto an array, or by improved software and stimulation strategies that can manipulate the existing electronics to provide "virtual" phosphenes by techniques such as current steering. Another area that is likely to provide significant benefits to future-generation prostheses is the use of vision processing algorithms that will optimize the electrical input to devices, thus maximizing potential outcomes.

Future studies of vision prostheses are also likely to investigate the use of devices in patients with earlier eye disease and more residual baseline vision. In these cases, prosthesis designs such as the suprachoroidal implant will have a significant benefit as they will not block the residual vision; hence, the device may add to patients' visual function. In comparison, epiretinal and subretinal devices will provide a physical barrier to the transmission of light to the remaining photoreceptors.

There is also interest as to whether vision prostheses could be beneficial in patients with congenital blindness. At present, all trial participants have had a history of formed vision to ensure that the visual pathways have formed prior to their vision loss. However, if the devices could be used in those with unformed visual pathways, and cause neural remapping of the pathway, then the potential population for this intervention would be increased.

It is an exciting time in the field of vision prostheses, with significant improvements in technology and patient outcomes likely in the coming years, leading to the chance of sight restoration for thousands of people worldwide.

\section{Acknowledgments}

Our research has been supported by the Australian Research Council (ARC) through its Special Research Initiative (SRI) in Bionic Vision Science and Technology grant to Bionic Vision Australia (BVA), 2010-2015. Centre for Eye Research Australia (CERA) receives Operational Infrastructure Support from the Victorian Government and is supported by National Health and Medical Research Council of Australia (NHMRC) Centre for Clinical Research Excellence Award 529923.

\section{Disclosure}

The authors report no conflicts of interest in this work.

\section{References}

1. World Health Organization. Fact Sheet: Visual Impairment and Blindness. Geneva: World Health Organization; 2014.

2. Foerster O. Beitraege zur Pathophysiologie der Sehbahn und der Sehsphaere [Contributions to the pathophysiology of the visual pathway and the visual sphere]. J Psychol Neurol. 1929;39:435-463. German.

3. Ho AC, Humayun MS, Dorn JD, et al. Long-term results from an epiretinal prosthesis to restore sight to the blind. Ophthalmology. 2015;122(8):1547-1554.

4. Kitiratschky VB, Stingl K, Wilhelm B, et al. Safety evaluation of "retina implant alpha IMS" - a prospective clinical trial. Graefes Arch Clin Exp Ophthalmol. 2015;253(3):381-387.

5. Ayton LN, Blamey PJ, Guymer RH, et al. First-in-human trial of a novel suprachoroidal retinal prosthesis. PLoS One. 2014;9(12):e115239. 
6. Fujikado T, Kamei M, Sakaguchi H, et al. Clinical trial of chronic implantation of suprachroidal-transretinal stimulation system for retinal prosthesis. Sensor Mater. 2012;24(4):181-187.

7. Brelen ME, Vince V, Gerard B, Veraart C, Delbeke J. Measurement of evoked potentials after electrical stimulation of the human optic nerve. Invest Ophthalmol Vis Sci. 2010;51(10):5351-5355.

8. Srivastava NR, Troyk PR. Some solutions to technical hurdles for developing a practical intracortical visual prosthesis device. Conf Proc IEEE Eng Med Biol Soc. 2006;1:2936-2939.

9. Weiland JD, Humayun MS, Dagnelie G, de Juan E Jr, Greenberg RJ, Iliff NT. Understanding the origin of visual percepts elicited by electrical stimulation of the human retina. Graefes Arch Clin Exp Ophthalmol. 1999;237(12):1007-1013.

10. Shivdasani MN, Sinclair NC, Dimitrov PN, et al. Factors affecting perceptual thresholds in a suprachoroidal retinal prosthesis. Invest Ophthalmol Vis Sci. 2014;55(10):6467-6481.

11. Horsager A, Boynton GM, Greenberg RJ, Fine I. Temporal interactions during paired-electrode stimulation in two retinal prosthesis subjects. Invest Ophthalmol Vis Sci. 2011;52(1):549-557.

12. Humayun MS, de Juan E Jr, Weiland JD, et al. Pattern electrical stimulation of the human retina. Vision Res. 1999;39(15):2569-2576.

13. Nau A, Bach M, Fisher C. Clinical tests of ultra-low vision used to evaluate rudimentary visual perceptions enabled by the BrainPort vision device. Transl Vis Sci Technol. 2013;2(3):1-12.

14. Stingl K, Bartz-Schmidt KU, Besch D, et al. Subretinal visual implant alpha IMS - clinical trial interim report. Vision Res. 2015;111(Pt B): 149-160.

15. da Cruz L, Coley BF, Dorn J, et al. The Argus II epiretinal prosthesis system allows letter and word reading and long-term function in patients with profound vision loss. Br J Ophthalmol. 2013(5):632-636.

16. Stingl K, Bartz-Schmidt KU, Gekeler F, Kusnyerik A, Sachs H, Zrenner E. Functional outcome in subretinal electronic implants depends on foveal eccentricity. Invest Ophthalmol Vis Sci. 2013;54(12):7658-7665.

17. Peachey NS, Chow AY. Subretinal implantation of semiconductorbased photodiodes: progress and challenges. J Rehabil Res Dev. 1999;36(4):371-376.

18. Humayun MS, Dorn JD, da Cruz L, et al. Interim results from the international trial of Second Sight's visual prosthesis. Ophthalmology. 2012;119(4):779-788.

19. Liew G, Michaelides M, Bunce C. A comparison of the causes of blindness certifications in England and Wales in working age adults (16-64 years), 1999-2000 with 2009-2010. BMJ Open. 2014;4:e004015.

20. Stone JL, Barlow WE, Humayun MS, de Juan E Jr, Milam AH. Morphometric analysis of macular photoreceptors and ganglion cells in retinas with retinitis pigmentosa. Arch Ophthalmol. 1992;110(11):1634-1639.

21. Humayun MS, Prince M, de Juan E Jr, et al. Morphometric analysis of the extramacular retina from postmortem eyes with retinitis pigmentosa. Invest Ophthalmol Vis Sci. 1999;40(1):143-148.

22. Ahuja AK, Yeoh J, Dorn JD, et al. Factors Affecting perceptual threshold in Argus II retinal prosthesis subjects. Transl Vis Sci Technol. 2013;2(4):1.

23. Zrenner E. Fighting blindness with microelectronics. Sci Transl Med. 2013;5(210):210ps16.

24. Weiland JD, Cho AK, Humayun MS. Retinal prostheses: current clinical results and future needs. Ophthalmology. 2011;118(11):2227-2237.

25. Hadjinicolaou AE, Meffin H, Maturana MI, Cloherty SL, Ibbotson MR. Prosthetic vision: devices, patient outcomes and retinal research. Clin Exp Optom. 2015;98(5):395-410.

26. Humayun MS, Dorn JD, Ahuja AK, et al. Preliminary 6 month results from the Argus II epiretinal prosthesis feasibility study. Conf Proc IEEE Eng Med Biol Soc. 2009:4566-4568.

27. Geruschat DR, Flax M, Tanna N, et al. FLORA ${ }^{\mathrm{TM}}$ : Phase I development of a functional vision assessment for prosthetic vision users. Clin Exp Optom. 2015;98:342-347.
28. Stett A, Barth W, Weiss S, Haemmerle H, Zrenner E. Electrical multisite stimulation of the isolated chicken retina. Vision Res. 2000;40(13): 1785-1795.

29. Luo YH, da Cruz L. A review and update on the current status of retinal prostheses (bionic eye). Br Med Bull. 2014;109:31-44.

30. Zrenner E, Bartz-Schmidt KU, Benav H, et al. Subretinal electronic chips allow blind patients to read letters and combine them to words. Proc Biol Sci. 2010;278(1711):1489-1497.

31. Stingl K, Bartz-Schmidt KU, Besch D, et al. Artificial vision with wirelessly powered subretinal electronic implant alpha-IMS. Proc Biol Sci. 2013;280(1757):20130077.

32. Keseru M, Feucht M, Bornfeld N, et al. Acute electrical stimulation of the human retina with an epiretinal electrode array. Acta Ophthalmol. 2012;90(1):e1-e8.

33. Richard G, Keseru M, Feucht M, Post N, Hornig R. Visual perception after long-term implantation of a retinal implant. Invest Ophthalmol Vis Sci. 2008;49:1786.

34. Nayagam DA, Williams RA, Allen PJ, et al. Chronic electrical stimulation with a suprachoroidal retinal prosthesis: a preclinical safety and efficacy study. PLoS One. 2014;9(5):e97182.

35. Villalobos J, Fallon JB, Nayagam DA, et al. Cortical activation following chronic passive implantation of a wide-field suprachoroidal retinal prosthesis. J Neural Eng. 2014;11(4):046017.

36. Nishida K, Sakaguchi H, Kamei M, et al. Visual sensation by electrical stimulation using a new direct optic nerve electrode device. Brain Stimul. 2015;8(3):678-681.

37. Veraart C, Wanet-Defalque MC, Gerard B, Vanlierde A, Delbeke J. Pattern recognition with the optic nerve visual prosthesis. Artif Organs. 2003;27(11):996-1004.

38. Löwenstein K, Borchardt M. Symptomatologie und elektrische Reizung bei einer Schußverletzung des Hinterhauptlappens [Symptomatology and electrical stimulation with a gunshot wound of the occipital lobe]. Deutsch Z Nervenheilkd. 1918;58:264-292. German.

39. Brindley GS, Lewin WS. The sensations produced by electrical stimulation of the visual cortex. J Physiol. 1968;196(2):479-493.

40. Dobelle WH. Artificial vision for the blind by connecting a television camera to the visual cortex. ASAIO J. 2000;46(1):3-9.

41. Merabet LB, Rizzo JF, Amedi A, Somers DC, Pascual-Leone A. What blindness can tell us about seeing again: merging neuroplasticity and neuroprostheses. Nat Rev Neurosci. 2005;6(1):71-77.

42. Born RT, Trott AR, Hartmann TS. Cortical magnification plus cortical plasticity equals vision? Vision Res. 2015;111(Pt B):161-169.

43. Schira MM, Wade AR, Tyler CW. Two-dimensional mapping of the central and parafoveal visual field to human visual cortex. J Neurophysiol. 2007;97(6):4284-4295.

44. Tootell RB, Hadjikhani NK, Vanduffel W, et al. Functional analysis of primary visual cortex (V1) in humans. Proc Natl Acad Sci US A. 1998;95(3):811-817.

45. Lewis PM, Ackland HM, Lowery AJ, Rosenfeld JV. Restoration of vision in blind individuals using bionic devices: A review with a focus on cortical visual prostheses. Brain Res. 2015;1595:51-73.

46. Wang C, Brunton E, Haghgooie S, Cassells K, Lowery A, Rajan R. Characteristics of electrode impedance and stimulation efficacy of a chronic cortical implant using novel annulus electrodes in rat motor cortex. J Neural Eng. 2013;10(4):046010.

47. Lepri BP. Is acuity enough? Other considerations in clinical investigations of visual prostheses. J Neural Eng. 2009;6(3):035003.

48. US Food and Drug Administration. Investigational Device Exemption (IDE) Guidance for Retinal Prostheses. Silver Spring, MD: US Food and Drug Administration; 2013.

49. Rizzo JF, 3rd, Ayton LN. Psychophysical testing of visual prosthetic devices: a call to establish a multi-national joint task force. $J$ Neural Eng. 2014;11(2):020301.

50. Chen SC, Suaning GJ, Morley JW, Lovell NH. Simulating prosthetic vision: I. Visual models of phosphenes. Vision Res. 2009;49(12): 1493-1506. 
Eye and Brain

\section{Publish your work in this journal}

Eye and Brain is an international, peer-reviewed, open access journal focusing on clinical and experimental research in the field of neuroophthalmology. All aspects of patient care are addressed within the journal as well as basic research. Papers covering original research, basic science, clinical and epidemiological studies, reviews and evaluations,

Submit your manuscript here: http://www.dovepress.com/eye-and-brain-journal

\section{Dovepress}

guidelines, expert opinion and commentary, case reports and extended reports are welcome. The manuscript management system is completely online and includes a very quick and fair peer-review system, which is all easy to use. Visit http://www.dovepress.com/testimonials.php to read real quotes from published authors. 